
increasing age http://ow.ly/tybI4

Michiru Sawahata ${ }^{1}$, Yukihiko Sugiyama ${ }^{1}$, Yosikazu Nakamura ${ }^{2}$, Masayuki Nakayama ${ }^{1}$, Naoko Mato ${ }^{1}$, Hideaki Yamasawa ${ }^{1}$ and Masashi Bando ${ }^{1}$

${ }^{1}$ Division of Pulmonary Medicine, Dept of Medicine, Jichi Medical University, Shimotsuke, Tochigi, and ${ }^{2}$ Dept of Public Health, Jichi Medical University, Shimotsuke, Tochigi, Japan.

Correspondence: M. Sawahata, Division of Pulmonary Medicine, Jichi Medical University, 3311-1 Yakushiji, Shimotsukeshi, Tochigi 329-0498, Japan. E-mail: d1114@jichi.ac.jp

Received: Jan 082014 | Accepted after revision: Jan 312014 | First published online: March 132014

Support statement: This study was partly supported by a grant to the Diffuse Lung Diseases Research Group from the Ministry of Health, Labour and Welfare, Japan (grant number H23-Nanchi-Ippan-023). M. Sawahata thanks the Japanese Society of Sarcoidosis and Other Granulomatous Disorders for awarding the Grant for Young Investigators (2013), and Jichi Medical University for awarding the JMU Graduate Student Start-Up Grant for Young Investigators (2012).

Conflict of interest: Disclosures can be found alongside the online version of this article at www.erj.ersjournals.com

\title{
References
}

Statement on sarcoidosis. Am J Respir Crit Care Med 1999; 160: 736-755.

Hillerdal G, Nöu E, Osterman K, et al. Sarcoidosis: epidemiology and prognosis. A 15-year European study. Am Rev Respir Dis 1984; 130: 29-32.

Morimoto T, Azuma A, Abe S, et al. Epidemiology of sarcoidosis in Japan. Eur Respir J 2008; 31: 372-379. [Japanese Society of Sarcoidosis and Other Granulomatous Disorders (JSSOG) diagnostic criteria and guidelines for sarcoidosis - 2006.]. Jpn J Sarcoidosis Other Granulomatous Disorders 2007; 22: 89-101.

5 Scadding JG. Prognosis of intrathoracic sarcoidosis in England. A review of 136 cases after five years' observation. Br Med J 1961; 2: 1165-1172.

6 Müller-Quernheim J. Sarcoidosis: immunopathogenetic concepts and their clinical application. Eur Respir J 1998; 12: 716-738.

7 Corrin B, Nicholson AG, Dewar A. Pathology. In: Mitchell D, Wells A, Spiro S, et al, eds. Sarcoidosis. London, Hodder Arnold, 2012; pp. 41-47.

$8 \quad$ Valeyre D, Prasse A, Nunes H, et al. Sarcoidosis. Lancet 2014; 383: 1155-1167.

9 Negi M, Takemura T, Guzman J, et al. Localization of Propionibacterium acnes in granulomas supports a possible etiologic link between sarcoidosis and the bacterium. Mod Pathol 2012; 25: 1284-1297.

10 Zaba LC, Smith GP, Sanchez M, et al. Dendritic cells in the pathogenesis of sarcoidosis. Am J Respir Cell Mol Biol 2010; 42: 32-39.

11 Linton PJ, Haynes L, Tsui L, et al. From nave to effector-alterations with aging. Immunol Rev 1997; 160: 9-18.

12 Sharma S, Dominguez AL, Lustgarten J. High accumulation of T regulatory cells prevents the activation of immune responses in aged animals. J Immunol 2006; 177: 8348-8355.

13 Shevach EM. Mechanisms of Foxp $3^{+}$T regulatory cell-mediated suppression. Immunity 2009; 30: 636-645.

14 Miyara M, Amoura Z, Parizot C, et al. The immune paradox of sarcoidosis and regulatory T cells. J Exp Med 2006; 203: $359-370$.

\section{Whole-body magnetic resonance imaging in extrathoracic sarcoidosis}

\author{
To the Editor:
}

The course of sarcoidosis is heterogeneous, and the assessment of pulmonary and extrathoracic organ involvement is important for clinical treatment decisions [1]. Whole-body imaging techniques have been evaluated to assess total disease activity $[2,3] .{ }^{18} \mathrm{~F}$-fluoro-2-deoxy-D-glucose positron emission tomography $\left({ }^{18} \mathrm{FDG}-\mathrm{PET}\right)$ and ${ }^{18} \mathrm{FDG}-\mathrm{PET} / \mathrm{computed}$ tomography (CT) allow a complete picture of active intra- and extrapulmonary sites [4]. Whole-body magnetic resonance imaging (WB-MRI) is an established diagnostic tool for multifocal disorders such as multiple myeloma and metastatic diseases [5, 6]. The role of WB-MRI in the assessment of extrathoracic organ involvement in patients with sarcoidosis has not yet been studied.

We present an institutional review board-approved study including 24 patients with histologically confirmed sarcoidosis. Patients were recruited regardless of treatment, apparent extrapulmonary involvement and symptoms; written informed consent was obtained. To estimate extrapulmonary disease 


\begin{tabular}{lc}
\hline a) Subjects & 24 \\
Age years & $50(28-76)$ \\
Females/males & $12 / 12$ \\
Race white/black/Asian/other & $23 / 1 / 0 / 0$ \\
Sarcoidosis-specific medication & \\
Corticosteroids only & $1(4)$ \\
Immunomodulator only & $3(13)$ \\
Corticosteroid+immunomodulator & $9(37)$ \\
$\quad$ None & $11(46)$ \\
Pulmonary function test & \\
FVC \% predicted & $92.2(55-106)$ \\
TLC \% predicted & $93.0(65-120)$ \\
FEV1\% predicted & $88.6(53-116)$ \\
DLCo \% predicted & $76.5(20-111)$ \\
ACE U.L-1 & $36.5(3-97)$ \\
sIL-2R pg-mL-1 & $445(113-1069)$ \\
ePOST & $13.2(1-32)$ \\
Patients with abnormal WB-MRI findings & \\
Skeletal system confidence score $\geqslant 1$ & $5(21)$ \\
Muscular system confidence score $\geqslant 1$ & $3(13)$ \\
Central nervous system confidence score $\geqslant 1$ & $1(4)$ \\
\hline
\end{tabular}
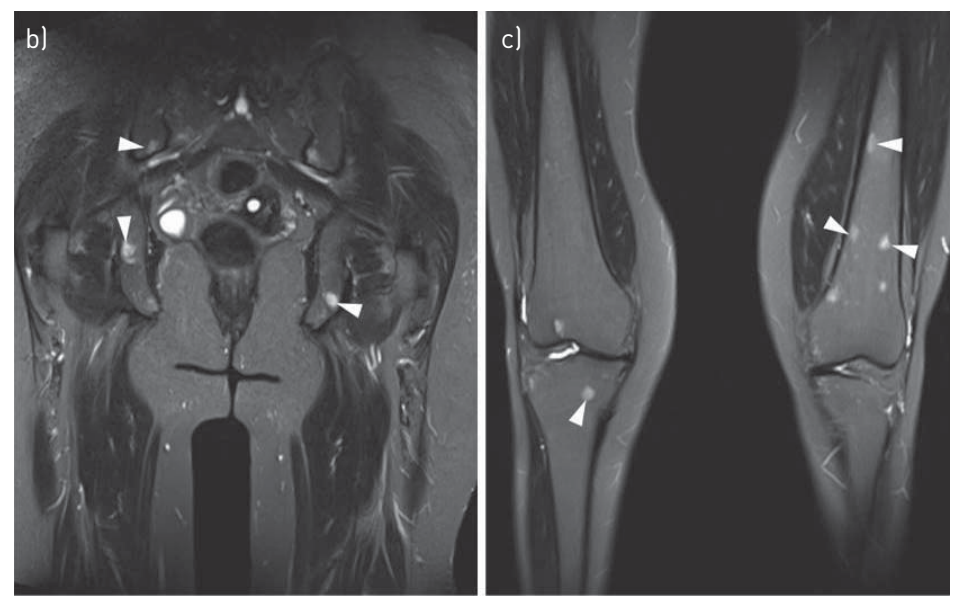

FIGURE 1 a) Baseline demographics, medication, pulmonary function, levels of angiotensin-converting enzyme (ACE) (normal range 12-68 U. $\mathrm{L}^{-1}$ ) and soluble interleukin-2 receptor (sIL-2R) (normal range $<477 \mathrm{pg} \cdot \mathrm{mL}^{-1}$ ), extrapulmonary physician organ severity tool (ePOST), and whole-body magnetic resonance imaging (WB-MRI) findings of all 24 patients with sarcoidosis. Data are presented as n, mean (range) or n (\%). FVC: forced vital capacity; TLC: total lung capacity; FEV1: forced expiratory volume in 1 s; DLCO: diffusing capacity of the lung for carbon monoxide. Portions of coronal WB-MRI (Short Tau Inversion Recovery images) show b) multiple osseous lesions probably related to sarcoidosis in the pelvis (arrowheads) and c) around both knees (arrowheads) in a 53-yearold female.

activity, the extrapulmonary physician organ severity tool (ePOST) was employed [7], scoring 17 organs (0: not affected; 6: very severely affected). Pulmonary function tests (PFTs) and serum levels of angiotensinconverting enzyme (ACE) and soluble interleukin-2 receptor (sIL-2R) were assessed. All subjects underwent WB-MRI on a clinical 1.5-T whole-body scanner with an 18-channel coil array system (Magnetom Avanto (Tim); Siemens Medical Solutions, Erlangen, Germany). The acquisition consisted of the following pulse sequences: coronal and sagittal T1-weighted Turbo Spin Echo (T1w) (repetition time (TR) $682 \mathrm{~ms}$ and echo time (TE) $11 \mathrm{~ms}$ ) and Short Tau Inversion Recovery (STIR) (TR $9630 \mathrm{~ms}$, TE $87 \mathrm{~ms}$ and inversion time $180 \mathrm{~ms}$ ) (slice thickness $5 \mathrm{~mm}$, intersection gap 20\% coronal and $10 \%$ sagittal, and field of view $50 \times 50 \mathrm{~cm}$ ), with composition of multiple image stacks to achieve coverage from head to pelvic floor for T1w and from head to feet for STIR sequences. No intravenous contrast material was administered. Images were read by two radiologists who were blinded to the clinical and laboratory data. Analysed compartments included the skeleton, muscles, abdominal viscera and the central nervous system (CNS). For each finding, a three-point confidence score was provided: probably related (score: 2), possibly related (score: 1) and unlikely to be related (score: 0 ). A lesion was classified as "probably related" when the appearance was consistent with sarcoidosis and other entities were unlikely. Lesions consistent with sarcoidosis, but having at least one equally likely cause, were classified as "possibly related". A lesion unlikely to be related to sarcoidosis was, by its location or morphology, typical of other entities (e.g. cysts related to osteoarthritis). In the skeletal system, bone lesions were defined as areas of oedema-like bone marrow signal abnormalities or cystic changes. In the muscular system, the muscles were evaluated for diffuse myopathic changes and nodular lesions. In the CNS, nodular or mass-like findings of the subcortical and periventricular white matter, the hypothalamic-pituitary axis, the spinal cord and the cauda equina were recorded. In the abdominal viscera, cystic, nodular or infiltrative changes of the liver, spleen and kidneys were recorded. The frequencies of WB-MRI findings were noted and evaluated for a cut-off level $\geqslant 1$. The one-tailed unpaired t-test was used to test for differences in laboratory data between patients with and without sarcoidosisrelated MRI findings. The differences in the frequencies of MRI findings (confidence score $\geqslant 1$ ) between patients with normal and abnormal laboratory tests and PFTs were tested by a two-tailed Fisher's exact test. All statistical tests were performed with SigmaPlot 12 (Scientific Solution SA, Pully, Switzerland). p-values $<0.05$ were considered significant.

Demographic and clinical characteristics of all patients and a summary of the WB-MRI findings are summarised in figure 1a. In seven (29\%) patients, increased serum levels of sIL-2R were measured, but all patients had normal serum ACE.

In nine $(38 \%)$ out of 24 patients, sarcoid lesions with confidence scores $\geqslant 1$ were noticed. Five $(21 \%)$ out of 24 patients had skeletal lesions with a confidence score $\geqslant 1$. One patient had a diffuse nodular osseous 
manifestation (fig. $1 \mathrm{~b}$ and c). Three (13\%) out of 24 patients had muscular findings of confidence score $\geqslant 1$. One patient showed massive nodular thickening of the cauda equina nerve roots. Brain white matter lesions, mostly subcortical, were noticed in $16(67 \%)$ out of 24 of patients, but were considered of confidence score 0 . No nodular or infiltrative changes of the liver, spleen or kidneys were noticed. Incidental findings requiring further work-up were seen in two patients: one patient had a sphenoidal wing meningioma and one patient had a lesion in the femur, which showed characteristic findings of an enchondroma on radiographs.

Patients with abnormal WB-MRI findings had significantly higher ePOST scores $(17.3$ versus $10.6, \mathrm{p}<0.05)$. There was no significant difference in ACE or sIL-2R levels between patients with and without sarcoidosis lesions of confidence $\geqslant 1$ on WB-MRI. The prevalence of skeletal findings was significantly higher in patients with reduced total lung capacity (TLC) $(<80 \%$ predicted) $(\mathrm{p}<0.02)$, in patients with reduced forced vital capacity $(\mathrm{FVC})(<80 \%$ predicted $)(\mathrm{p}<0.02)$ or in patients with reduced diffusing capacity of the lung for carbon monoxide (DLCO) $(<60 \%$ predicted) $(\mathrm{p}<0.01)$. TLC, FVC and DLCO \% pred were significantly lower in those patients with sarcoidosis-compatible skeletal lesions compared with those patients without skeletal abnormalities.

We investigated the utility of WB-MRI with regard to the assessment of extrathoracic organ involvement in patients with sarcoidosis. By using WB-MRI, extrathoracic organ involvement was detected in $38 \%$ of this nonselected patient group and this is in accordance with data obtained by PET/CT [8]. In agreement with previous PET studies $[4,8,9]$, WB-MRI revealed the skeleton to be the most frequent site of extrathoracic involvement. Sarcoid muscular lesions were found in $13 \%$ of patients, supporting the assumption that muscular involvement is more frequent than clinically expected [10].

Sarcoidal involvement of the CNS was detected in one patient; however, without contrast administration, the sensitivity is significantly reduced. Nonspecific cerebral white matter changes can be commonly found on magnetic resonance images of asymptomatic healthy individuals [11].

Patients with WB-MRI changes potentially related to sarcoidosis had significantly higher ePOST scores, which confirmed a higher extrapulmonary disease activity. We found no correlation between WB-MRI positivity and serological markers, and this is in accordance with previous studies $[2,3]$. Finally, patients with abnormal skeletal findings had lower lung volumes than patients with inconspicuous WB-MRI. This argues for a more pronounced functional impairment and, hence, more active pulmonary disease in this group. The detection of asymptomatic musculoskeletal sarcoid manifestations at WB-MRI can alter the estimation of the overall granulomatous load and disease activity, and together with the demonstrated functional impairment in patients with WB-MRI positivity, it might be speculated that WB-MRI represents a marker of total disease activity. Our findings complete previous data that demonstrated the value of PET/ CT for the assessment of disease activity in sarcoidosis [2-4,9], with the advantage of MRI being radiation free. Recent data suggested that PET is a predictor of pulmonary function in sarcoidosis [12]; however, the radiation exposure of PET should not be underestimated and PET is not recommended for standard workup procedures [13].

We acknowledge that even though WB-MRI findings assessed as being possibly related to sarcoidosis are highly suggestive of sarcoidosis, definite confirmation would require tissue biopsy. To keep the scan-time within a reasonable frame, no contrast administration was performed, which reduced the sensitivity for the detection of sarcoidal lesions, particularly of cardiac and CNS involvement. The spatial resolution of WBMRI is lower than in focused MRI (e.g. cardiac imaging) and, together with breathing-motion artefacts, this might have precluded the detection of small lesions. Finally, negative WB-MRI scans do not rule out organ involvement.

In summary, we suggest that WB-MRI is a useful technique to depict extrathoracic organ involvement in patients with sarcoidosis and might therefore be a valid tool to assess extrathoracic disease activity. Therapeutic guidelines account for extensive lung disease, involvement of the heart, eye or CNS [14]. Furthermore, subgroups of patients with predominantly extrapulmonary sarcoidosis seem to profit more from particular treatments [15]. However, the question whether patients with additional, more diffuse extrathoracic disease have a more severe course and should be treated more aggressively has not been elucidated yet. We hypothesise that the detection of additional musculoskeletal disease by WB-MRI may influence therapeutic decisions. Further studies are needed to substantiate the role of WB-MRI in the diagnostic and prognostic work-up of sarcoidosis patients. 
Katrin E. Hostettler ${ }^{1,3}$, Vlad A. Bratu ${ }^{2,3}$, Arne Fischmann ${ }^{2}$, Michael Tamm ${ }^{1}$ and Ulrich Studler ${ }^{2}$

${ }^{1}$ Clinic of Respiratory Medicine, University Hospital Basel, Basel, and ${ }^{2}$ Dept of Radiology, University Hospital Basel, Basel, Switzerland. ${ }^{3}$ Both authors contributed equally.

Correspondence: K.E. Hostettler, Clinic of Respiratory Medicine, University Hospital Basel, Petersgraben 4, 4031 Basel, Switzerland. E-mail: katrin.hostettler@usb.ch

Received: Oct 252013 | Accepted after revision: Feb 122014 | First published online: March 132014

Support statement: This work was supported by a nonconditional grant from the Gottfried and Julia Bangerter-RhynerStiftung. The funders had no role in study design, data collection and analysis, decision to publish, or preparation of the manuscript.

Conflict of interest: Disclosures can be found alongside the online version of this article at www.erj.ersjournals.com

\section{References}

Judson MA, Baughman RP, Teirstein AS, et al. Defining organ involvement in sarcoidosis: the ACCESS proposed instrument. Sarcoidosis Vasc Diffuse Lung Dis 1999; 16: 75-86.

2 Teirstein AS, Machac J, Almeida O, et al. Results of 188 whole-body fluorodeoxyglucose positron emission tomography scans in 137 patients with sarcoidosis. Chest 2007; 132: 1949-1953.

3 Keijsers RG, Verzijlbergen FJ, Oyen WJ, et al. ${ }^{18} \mathrm{~F}-\mathrm{FDG}$ PET, genotype-corrected ACE and sIL-2R in newly diagnosed sarcoidosis. Eur J Nucl Med Mol Imaging 2009; 36: 1131-1137.

4 Ambrosini V, Zompatori M, Fasano L, et al. ${ }^{18} \mathrm{~F}-\mathrm{FDG}$ PET/CT for the assessment of disease extension and activity in patients with sarcoidosis: results of a preliminary prospective study. Clin Nucl Med 2013; 38: e171-e177.

5 Lauenstein TC, Goehde SC, Herborn CU, et al. Whole-body MR imaging: evaluation of patients for metastases. Radiology 2004; 233: 139-148.

6 Dutoit JC, Vanderkerken MA, Verstraete KL. Value of whole body MRI and dynamic contrast enhanced MRI in the diagnosis, follow-up and evaluation of disease activity and extent in multiple myeloma. Eur J Radiol 2013; 82: 1444-1452.

7 Judson MA, Baughman RP, Costabel U, et al. Efficacy of infliximab in extrapulmonary sarcoidosis: results from a randomised trial. Eur Respir J 2008; 31: 1189-1196.

8 Sobic-Saranovic D, Grozdic I, Videnovic-Ivanov J, et al. The utility of ${ }^{18}$ F-FDG PET/CT for diagnosis and adjustment of therapy in patients with active chronic sarcoidosis. J Nucl Med 2012; 53: 1543-1549.

9 Mostard RL, Voo S, van Kroonenburgh MJ, et al. Inflammatory activity assessment by F18 FDG-PET/CT in persistent symptomatic sarcoidosis. Respir Med 2011; 105: 1917-1924.

10 Otake S, Ishigaki T. Muscular sarcoidosis. Semin Musculoskelet Radiol 2001; 5: 167-170.

11 Hopkins RO, Beck CJ, Burnett DL, et al. Prevalence of white matter hyperintensities in a young healthy population. J Neuroimaging 2006; 16: 243-251.

12 Keijsers RG, Verzijlbergen EJ, van den Bosch JM, et al. ${ }^{18} \mathrm{~F}-\mathrm{FDG}$ PET as a predictor of pulmonary function in sarcoidosis. Sarcoidosis Vasc Diffuse Lung Dis 2011; 28: 123-129.

13 Keijsers RG, van den Heuvel DA, Grutters JC. Imaging the inflammatory activity of sarcoidosis. Eur Respir J 2013; 41: 743-751.

14 Iannuzzi MC, Fontana JR. Sarcoidosis: clinical presentation, immunopathogenesis, and therapeutics. JAMA 2011; 305: 391-399.

15 Hostettler KE, Studler U, Tamm M, et al. Long-term treatment with infliximab in patients with sarcoidosis. Respiration 2012; 83: 218-224. 